Journal of Mathematics and Informatics

Vol. 7, 2017, 87-94

ISSN: 2349-0632 (P), 2349-0640 (online)

Published 12 June 2017

www.researchmathsci.org

DOI: http://dx.doi.org/10.22457/jmi.v7al1

Journal of

Mathematics and

Informatics

\title{
Study on the Evaluation Method of Internet Economy
}

\section{Development}

\author{
Zi-fu Fan and Wen-qin Li
}

School of Economics and Management

Chongqing University of Posts and Telecommunications

Chongqing-400065, China

E-mail:1wqcoco@126.com

Received 16 May 2017; accepted 10 Jun 2017

Abstract. There are qualitative and quantitative evaluation indexes in the process of the Internet economy development evaluation. In this paper we analyze the shortcomings of the existing Internet economy development evaluation index systems, and give a new set of Internet economy development evaluation index. For the qualitative indicators of Internet economy, we use interval number and fuzzy number to describe uncertainty and fuzziness in the evaluation. For the quantitative indicators of Internet economy, we get evaluation value of real numbers by statistical approach. For hybrid evaluation data contain real number, interval number and fuzzy number, we propose an evaluating method of Internet economy development based on TOPSIS method.

Keywords: Internet economy; evaluation method; hybrid evaluation scale; TOPSIS

\section{AMS Mathematics Subject Classification (2010):15B15}

\section{Introduction}

The Internet economy is the sum total of economic activity based on the Internet [1]. At present, there is no uniform indicator system to measure the Internet economy development in the world. Only McKinsey released the IGDP index measuring the size of the Internet economy in 2014 [2]. Indicators that affect Internet economy development are not only qualitative evaluation indicators, but also quantitative evaluation indicators. The existing evaluation methods are based on the evaluation of quantitative evaluation index, while ignoring the application of qualitative evaluation in the evaluation process. The fact is that the qualitative and quantitative indicators are existing at the same time in the process of Internet economy evaluation. In order to better evaluate Internet economy 


\section{Zi-fu Fan and Wen-qin Li}

development and make evaluation results more scientific and reasonable, we must consider hybrid evaluation method includes quantitative and qualitative indicators. According to the actual situation, we will present a hybrid evaluation method of Internet economy development that includes qualitative and quantitative indicators.

\section{Indicator system of Internet economy development}

We should follow the comprehensive, practical, measurable and systematic selection principles in the process of indicator selection. It makes the indicators selected can comprehensively reflect all aspects of Internet economy development. And each indicator is convenient to collect and evaluate. For the Internet economy complex evaluation system, the corresponding evaluation indicator levels are also complex. According to arranging the listed evaluation indicators, paper will form the Internet economy development multi-level evaluation indicator system, as shown in Figure 1.

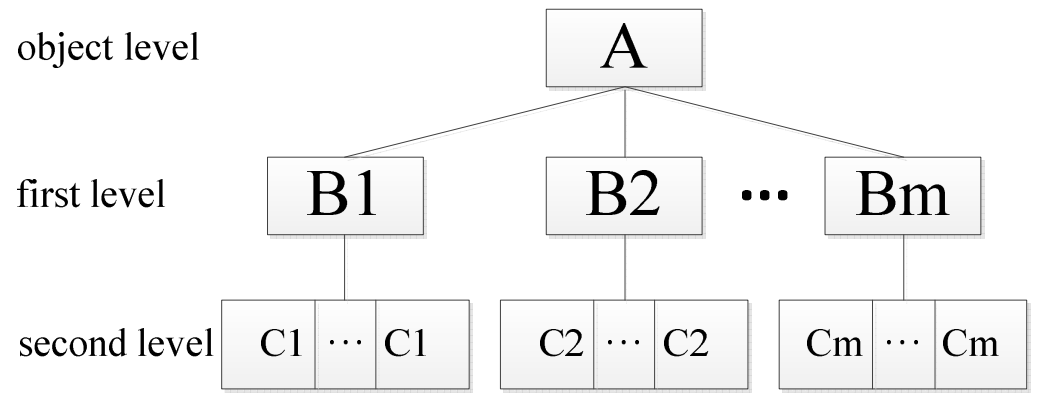

Figure 1: Multi-level indicator system of Internet economy development

Then, we summarize the research results of existing scholars to determine the final evaluation indicator set that affect Internet economy [3-6].

Economic base (B1): it means external economic base of Internet economy, evaluating from the aspects such as per capita GDP, employment rate, industry types and so on.

Change of industrial structure (B2):it means adjustment of industrial structure compared with previous cycle, evaluating from the aspects such as the construction of network serve, regional websites and so on.

Penetrative extent (B3): it means the effect of the Internet stimulated traditional industries, evaluating from the aspects such as the index of informatization and industrialization, the level of agricultural modernization.

Competitiveness level (B4): it's evaluated from the aspects such as the possession of the new generation of information technology industry, Internet financial transactions, E-commerce transactions and so on. 
Study on the Evaluation Method of Internet Economy Development

Sustainable level (B5): it's evaluated from the aspects such as Internet penetration, $R \& D$ expenditure, the influence of Internet industry on environment and so on.

In summary, paper determines the indicator system from five aspects: economic base, change of industrial structure, penetrative extent, competitiveness level and sustainable level. This indicator system contains all aspects of Internet economy development, so it fits the principles of indicator selection.

Before showing evaluation method of Internet economy development based on hybrid evaluation method, the following definitions and theorems are given:

Definition 1. Define continuous interval data's ordered weighted averaging operator (C-OWA) of the interval number $[\mathrm{a}, \mathrm{b}]$ as:

$$
f_{\rho}([a, b])=\int_{0}^{1} \frac{d \rho(y)}{d y}(b-y(b-a)) d y
$$

$\operatorname{Let} \rho(y)=y^{r}(r \geq 0)$, so C-OWA dependent on $[a, b] \operatorname{is} f_{\rho}([a, b])=\frac{b+r a}{r+1}$.

C-OWA can integrate all information of interval number $[a, b]$, and keep the uncertainty of interval number $[a, b]$ as far as possible.

Definition 2. Define triangular fuzzy number $\tilde{a}=\left(a_{l}, a_{m}, a_{r}\right)$ as:

$$
\mu_{\tilde{a}}(\mathrm{x})=\left\{\begin{array}{l}
\frac{x-a_{l}}{a_{m}-a_{l}} a_{l} \leq \mathrm{x} \leq a_{m} \\
\frac{a_{r}-x}{a_{r}-a_{m}} a_{m} \leq \mathrm{x} \leq a_{r}
\end{array}\right.
$$

where, $a_{l} \leq a_{m} \leq a_{r}$. If $a_{l}=a_{m}=a_{r}$, triangular fuzzy number will degenerate into common real number.

Definition 3. Define fuzzy continuous ordered weighted averaging operator (FC-OWA) of triangular fuzzy number $\tilde{a}=\left(a_{l}, a_{m}, a_{r}\right)$ as:

$$
F f_{\rho}(\tilde{a})=\int_{0}^{1}\left(\int_{0}^{1} \frac{d \rho(y)}{d y}\left(\tilde{a}_{l \alpha}-y\left(\tilde{a}_{l \alpha}-\tilde{a}_{r \alpha}\right)\right) d y\right) d \alpha
$$

where, $\left[\tilde{a}_{l \alpha}, \tilde{a}_{r \alpha}\right]$ is $\alpha$-cut set of triangular fuzzy number $\tilde{a}=\left(a_{l}, a_{m}, a_{r}\right)$. Let $\rho(y)=$ $y^{\lambda}(\lambda \geq 0)$, so FC-OWA dependent on

$$
\tilde{a}=\left(a_{l}, a_{m}, a_{r}\right) \text { is } F f_{\rho}(\tilde{a})=\frac{1}{2}\left(a_{m}+\frac{a_{r}+\lambda a_{l}}{\lambda+1}\right) .
$$

According to the principle of C-OWA can know, after FC-OWA converted triangular fuzzy number into real number, real number can contain the original fuzzy information of triangular fuzzy number. 


\section{Zi-fu Fan and Wen-qin Li}

\section{Hybrid evaluation method of Internet economy}

In the evaluation of level of Internet economy development, we find some quantitative indicators finally become real evaluation data by statistics analysis. But some qualitative indicators can be obtained only through expertise, because they are not easy to measure. Expert is erely on experts' evaluation habits, so some experts give interval-type evaluation data, some experts give language evaluation data. Normally, the language evaluation data is transformed into the form of triangular fuzzy number. Thus the hybrid evaluation data of real number, interval number and fuzzy number coexist will happen in the process of evaluation. We assume the level of Internet economy development in $\mathrm{n}$ regions need to evaluate, and invite experts in related fields to establish the expert group. For quantifiable indicators, we use the method of data statistics. For qualitative indicators, we comprehensively evaluate experts' personal opinions. Finally, the evaluation data are shown in Table 1.

Table 1: Evaluation information of $\mathrm{n}$ regional Internet economy development

\begin{tabular}{cccccc}
\hline & B1 & B2 & B3 & B4 & B5 \\
\hline region 1 & $x_{11}$ & $x_{12}$ & $x_{13}$ & $x_{14}$ & $x_{15}$ \\
region 2 & $x_{21}$ & $x_{22}$ & $x_{23}$ & $x_{24}$ & $x_{25}$ \\
$\ldots \ldots$ & $\ldots \ldots$ & $\ldots \ldots$ & $\ldots \ldots$ & $\ldots \ldots$ & $\ldots \ldots$ \\
region $\mathrm{n}$ & $x_{n 1}$ & $x_{n 2}$ & $x_{n 3}$ & $x_{n 4}$ & $x_{n 5}$ \\
\hline
\end{tabular}

where, $x_{i j}$ is the evaluation value of evaluating the $\mathrm{j}$-th index of the $\mathrm{i}$-th area. And $x_{i j}$ is one of the evaluation scale of real number, interval number and triangular fuzzy number.

For the evaluation matrix of real number, interval number and fuzzy number coexist, especially involving the calculation and comparison between the three scales, that will increase the difficulty of information handling. In order to simplify the difficulty of information handling and keep the uncertainty information of evaluation scale of interval number and fuzzy number as far as possible, we use C-OWA in definition 1 and FC-OWA in definition 3 to make information fusion of interval number and triangular fuzzy numbers in Table 1. The evaluation information after eliminating uncertainty by C-OWA and FC-OWA are shown in Table 2.

Table 2: Evaluation information after eliminating uncertainty

\begin{tabular}{cccccc}
\hline & $\mathrm{B} 1$ & $\mathrm{~B} 2$ & $\mathrm{~B} 3$ & $\mathrm{~B} 4$ & $\mathrm{~B} 5$ \\
\hline region 1 & $y_{11}$ & $y_{12}$ & $y_{13}$ & $y_{14}$ & $y_{15}$ \\
region 2 & $y_{21}$ & $y_{22}$ & $y_{23}$ & $y_{24}$ & $y_{25}$ \\
$\ldots \ldots$ & $\ldots \ldots$ & $\cdots \cdots$ & $\cdots \cdots$ & $\cdots \cdots$ & $\cdots \cdots$ \\
region n & $y_{n 1}$ & $y_{n 2}$ & $y_{n 3}$ & $y_{n 4}$ & $y_{n 5}$ \\
\hline
\end{tabular}


Study on the Evaluation Method of Internet Economy Development

where, $y_{i j}$ is the evaluation value of evaluating the $\mathrm{j}$-th index of the $\mathrm{i}$-th area after using C-OWA and FC-OWA to eliminate uncertainty. And $y_{i j}$ is real number.

In the process of evaluation on Internet economy, there are benefit and cost type indicators. So we need to uniform dimension of evaluation indicators, common standardization as follows:

For benefit type indicator: $r_{i j}=\frac{y_{i j}}{\max \left\{y_{i j}\right\}}, i=1,2, \cdots, n$

For cost type indicator: $r_{i j}=\frac{\min \left\{y_{i j}\right\}}{y_{i j}}, i=1,2, \cdots, n$

Set processed evaluation information as follows:

Table 3: Evaluation information after uniform dimension

\begin{tabular}{cccccc}
\hline & $\mathrm{B} 1$ & $\mathrm{~B} 2$ & $\mathrm{~B} 3$ & $\mathrm{~B} 4$ & $\mathrm{~B} 5$ \\
\hline region 1 & $r_{11}$ & $r_{12}$ & $r_{13}$ & $r_{14}$ & $r_{15}$ \\
region 2 & $r_{21}$ & $r_{22}$ & $r_{23}$ & $r_{24}$ & $r_{25}$ \\
$\ldots \ldots$ & $\ldots \ldots$ & $\ldots \ldots$ & $\ldots \ldots$ & $\ldots \ldots$ & $\ldots \ldots$ \\
region $\mathrm{n}$ & $r_{n 1}$ & $r_{n 2}$ & $r_{n 3}$ & $r_{n 4}$ & $r_{n 5}$ \\
\hline
\end{tabular}

Considering the influence degree of each indicator on Internet economy is not the same in the process of evaluation on Internet economy. So we must consider influence of each indicator on Internet economy. Set the weight vector of 5 evaluation indicators through combination weighting as:

$$
\mathrm{W}=\left(w_{1}, w_{2}, w_{3}, w_{4}, w_{5}\right)
$$

After identifying the evaluation scale and the index weight vector, we get the evaluation information. Finally, we need to make evaluation information fused to get the final evaluation result of regional Internet economic development. TOPSIS is a common multi-attribute evaluation method, and it achieves the sorting and evaluation of Internet economy development by measuring the close degree between evaluation vector of each region and the default of the positive and negative ideal state. We usually choose all the evaluation data of the maximum and the minimum when we have choices of the positive and negative ideal state [7-8].

The positive ideal state: $Z^{+}=\left(z_{1}^{+}, z_{2}^{+}, z_{3}^{+}, z_{4}^{+}, z_{5}^{+}\right)$

where: $z_{j}^{+}=\max \left\{r_{i j}\right\}, i=1,2, \cdots, n$

The negative ideal state: $Z^{-}=\left(z_{1}^{-}, z_{2}^{-}, z_{3}^{-}, z_{4}^{-}, z_{5}^{-}\right)$ where: $z_{j}^{-}=\min \left\{r_{i j}\right\}, i=1,2, \cdots, n$

After getting positive and negative ideal state, we calculate the distance between evaluation vector of $\mathrm{i}$-th regional Internet economy $r_{i}=\left(r_{i 1}, \cdots, r_{i 5}\right)$ and the positive negative ideal state.

The weighted distance of the positive ideal state as: 
Zi-fu Fan and Wen-qin Li

$$
d^{+}\left(r_{i}, Z^{+}\right)=\frac{\sum_{j=1}^{5} w_{j}^{2} r_{i j} z_{j}^{+}}{\sqrt{\sum_{j=1}^{5} w_{j}^{2} r_{i j}^{2}} \sqrt{\sum_{j=1}^{5} w_{j}^{2}\left(z_{j}^{+}\right)^{2}}}
$$

The weighted distance of the negative ideal state as:

$$
d^{-}\left(r_{i}, Z^{-}\right)=\frac{\sum_{j=1}^{5} w_{j}^{2} r_{i j} z_{j}^{-}}{\sqrt{\sum_{j=1}^{5} w_{j}^{2} r_{i j}^{2}} \sqrt{\sum_{j=1}^{5} w_{j}^{2}\left(z_{j}^{-}\right)^{2}}}
$$

The weighted similar degree of the final $r_{i}=\left(r_{i 1}, \cdots, r_{i 5}\right)$ and the ideal state as:

$$
\sigma_{i}=\frac{d^{-}\left(r_{i}, Z^{-}\right)}{d^{-}\left(r_{i}, Z^{-}\right)+d^{+}\left(r_{i}, Z^{+}\right)}
$$

The final optimal criterion is: the higher of $\sigma_{i}$, the higher the level of Internet economy development in the corresponding region.

\section{Empirical analysis}

The regular and effective evaluation of the level of regional Internet economy development is the main way to grasp the situation of regional Internet economy development and formulate the strategy of regional Internet economy development. In order to evaluate the Internet economy development of 5 counties in a province effectively, we invite relevant experts to evaluate the Internet economy development situation of 5 counties. The hybrid evaluation matrix as follows:

Table 4: Evaluation information of 5 counties' Internet economy

\begin{tabular}{lccccc}
\hline & B1 & B2 & B3 & B4 & B5 \\
\hline County 1 & $(7.9,8.1,8.6)$ & $(7.2,7.7,7.9)$ & 9.5 & {$[7.9,8.4]$} & {$[5.3,6.7]$} \\
County 2 & $(8.4,9.0,9.4)$ & $(5.0,5.4,6.1)$ & 9.7 & {$[9.0,9.5]$} & {$[7.5,8.1]$} \\
County 3 & $(5.7,6.2,6.7)$ & $(9.0,9.5,9.8)$ & 8.1 & {$[6.5,7.1]$} & {$[7.4,7.6]$} \\
County 4 & $(8.7,9.1,9.6)$ & $(8.9,9.6,9.8)$ & 6.4 & {$[5.3,5.9]$} & {$[8.1,8.9]$} \\
County 5 & $(5.9,6.3,6.5)$ & $(6.1,6.5,6.9)$ & 9.2 & {$[8.4,9.1]$} & {$[6.5,6.8]$} \\
\hline
\end{tabular}

$\operatorname{Let} \rho(y)=y^{4.5}$, we use C-OWA and FC-OWA to fuse information of evaluation data, and convert hybrid evaluation data that contains interval number, triangular fuzzy number and real number into real evaluation data. It's easy to evaluate or calculate, and evaluation information is shown in Table 5:

Table 5: Evaluation information expressed in real number

\begin{tabular}{cccccc}
\hline & B1 & B2 & B3 & B4 & B5 \\
\hline County 1 & 8.0636 & 7.5136 & 9.5000 & 7.9909 & 5.5538 \\
County 2 & 8.7909 & 5.3012 & 9.7000 & 9.0912 & 7.6091 \\
County 3 & 6.0398 & 9.3227 & 8.1000 & 6.6091 & 7.4364 \\
County 4 & 8.9820 & 9.3325 & 6.3000 & 5.4087 & 8.2466 \\
County 5 & 6.5636 & 6.3727 & 9.2000 & 8.5273 & 6.5545 \\
\hline
\end{tabular}


Study on the Evaluation Method of Internet Economy Development

The positive and negative ideal state of Internet economy development level of 5 counties as:

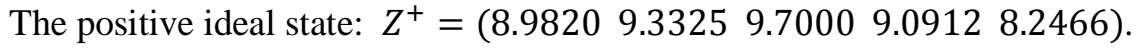

The negative ideal state: $Z^{-}=\left(\begin{array}{lllll}6.0398 & 5.3012 & 6.3000 & 5.4087 & 5.5538\end{array}\right)$.

Set the final weight vector of 5 evaluation indicators through combination weighting as:

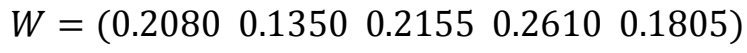

So the weighed distance between Internet economy development of each county and the positive and negative ideal state as:

Weighed distance of the positive ideal state:

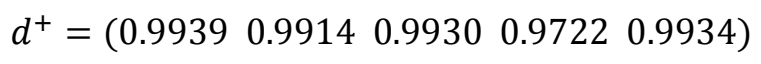

Weighed distance of the negative ideal state:

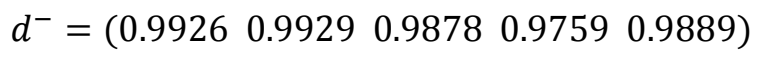

The weighted similar degree of Internet economy development of each county and the positive negative ideal state as:

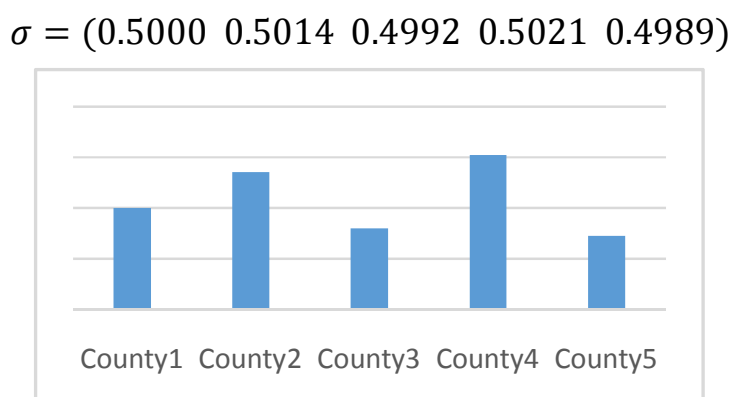

Figure 2: The weighted similar degree of 5 counties

So the sorting result of 5 counties' Internet economy development level as:

County $4>$ County $2>$ County $1>$ County $3>$ County 5

Through experts evaluated Internet economy development level of 5 counties, we can know Internet economy development level of county 4 is the best, Internet economy development level of county 5 is the worst. From the similar degree indicators, we can know Internet economy development of the five counties should be in the same level. By analysis, the main reason is the five counties are in the same province and face the similar opportunities and challenges. Finally, numeral example shows the practicality and effectiveness of the proposed method. The limitation is the design of the indicator system may need to be improved, expecting other scholars' future research.

Acknowledgements. This work is supported by the Humanities and Social Science Foundation of Education Department of Chongqing Province (No. 14SKF03). 


\section{Zi-fu Fan and Wen-qin Li}

\section{REFERENCES}

1. Yue Ma, Research on Internet economy valuation, Technology and Business, 1 (2016) 19-20.

2. Qiang-sen Hua, Gao-dun Ou and Jia-ming Liu, Chinese digital transformation: the impact of Internet on productivity and growth, McKinsey Global Institute, 2014.

3. Xian-wei Xue, Zong-xian Feng and Jian-qing Wang, Design of Chinese Internet economy level measurement index system, China Soft Science, 8 (2004) 51-59.

4. Y.Perfiliev, Development of the Internet in Russia, Eurasian Geography and Economics, 43(5) (2002) 411-421.

5. C Kenny, The Internet and Economic Growth in Less-developed Countries, Oxford Development Studies, 31(1) (2010) 99-113.

6. Li-chang Zhao, Internet economy and Chinese industrial transformation and upgrading, Contemporary Economy and Management, 12 (2015) 54-59.

7. Li-wen Chen, Quan-cheng Zheng and Zhi-kun Zhang, Evaluation of cargo loading reinforcement scheme based on hybrid TOPSIS, Traffic Science and Technology and Economy, 5( 2011) 67-69.

8. Shu-li Yan, Si-feng Liu and Jian Zhu, Decision-making method of TOPSIS based on entropy measure for three-parameter interval number information, Chinese Journal of Management Science, 6 (2013) 145-151. 